stimulates the kidneys and causes an inordinate and excessive secretion of urine, I have found this obstacle to its use removed by the administration of a dose of gallie acid for four or five successive nights.

Alihough the Sprudel comes to the surface at the temperature of boiling water, I have found it as useful and far more agreeable when taken cold.

I purposely refrain from any speculation on the existence of a materies morbi, in which, however, I have a firm belief; as well as from other theoretical questions. 'l'hese I shall reserve for a future occasion; but such are the facts.

\section{OVARIAN CYSTS.}

By John F. Nichorsos, F.R.C.S., Stratford Green.

As the treatment of ovarian cysts is of the deepest interest to the surgeon, I beg to send two cases which have occurred in my practice; and as they passed under the eyes of several of our London eminent men, they will, doubtless, deserve the greater attention.

CASE I. Jan. 17, 1861. I was called to see a lady, aged 42 , whom I had attended in six natural labours, and who had always been healthy. She had passed a resiless night; and awoke with great pain across the loins, shooting towards the pubes. The pain was constant, but had severe exacerbations. She said she could not exist much longer. There had been slight chilliness, but no rigor; and the weather was unusually cold. There was no fever; the tongue was clean and moist; the skin cool ; pulse under 80 ; and her face rather pale. Vomiting came on during the day, but only of the fluids taken, and having no trace of bile. Opiates and ether were given freely, with only very partial relief. The bowels had been moved during the morning; and the urine was free and natural. The seat of pain pointed to the kidneys, as if a calculus were passing along the ureter; but it did not extend into the thighs or pudenda. She was five months pregnant, and had felt the child distinctly in the morning.

Twelve leeches were applied to the lower portion of the abdomen on the right side, as she complained of more tenderness here; fomentations with turpentine epithems were freely applied, with chloroform, aconite, and tupentine, to the loins. The leeches gave ease; but she had no sleep; and on the 18th, the pain again returned, extending higher towards the umbilicus, with vomiting of everything swallowed. Iced drinks were taken, and one grain of calomel and opium every two hours. She could not raise herself in bed, owing to the excessive pain and tenderness, which were so great that the very slightest pressure was insupportable. Twelve Jeeches were a second time applied, and, as before, with some rolief.

Jan. 19 was passed with slight amendment; but in the evening she began to draw her legs up towards the ab. domen, which, below the umbilicus more especially, was exceedingly tender to the touch. Opiates were still given, but without procuring sleep, and only with very partial ease. The pulse had risen to 112 , but there was no fever; and the tongue was clean and moist. I now examined the os uteri; it was very high up, closed, and there was no discharge of any kind.

Jan. 20. Dr. $\longrightarrow$ met me in consultation. He agreed that there was decided peritonitis; but the cause was not clear. He suggested that there might be an extrauterine pregnancy, with rupture into Douglas's sac, accountilig for the pain commencing posteriorly in the back, and shooting thence towards the pubes and abdominal walls. He agreed that it was unusual to see peritonitis in pregnancy; and thought that there was some internal mischief of this kind. He advised the free exhibition of opiates, omitting the calomel; the abdomen to be covered with an ointment, composed of an ounce of the milder mercurial ointment, with two drachms of extract of belladonna; and effervescing salines with hydrocyanic acid, and beef tea, to be the form of nutriment. He thouglit that the acute stage was passed; and that by time and patience she would do well. She now lay constantly on her back, and the vomiting was incessant.

Jan. 24. All her symptoms were aggravated. The pulse was 130 to 140 . The eyes were sunken; the countenance very anxious. The opium had produced only two hours sleep, with dozes of a few minutes duration, disturbed by fits of pain. The latter had become less severe; but had extended gradually from below upwards over the whole abdomen, and was complained of most at the epigastrium. There was also distressing hiccup, and extreme tympanitis. The fœtus had not been felt for two days; but there was no discharge nor labourpain. She lay on her back, with her legs constantly. drawn up, but could bear to turn a little towards the right side. The urine was now scanty and high coloured. There were great exhaustion and sense of faintness. The nights were very restless, and the pain was always then increased. Another physician met me in consultation; and fully concurred that there was extensive peritonitis, but could not state the cause. He thought the case now hopeless, and advised tincture of opium and chloric ether as palliatives. An enema had been administered, as the bowels had not acted from the first, and was repeated. She said she could not strain to pass fæces. A pint of gruel, with an ounce of turpentine, gave but little pain; a very small motion passed with much flatus. She now grew weaker; the mind, though clear when roused, became vacant: the countenance sallow and anxious, with sunken eyeballs; the tongue dry and brown in the centre, with moist edges; vomiting and hiccup incessant; a dark brown bilious fluid was ejected with scarcely any effort; tympanitis so urgent that she said she should burst; and the pulse quick and thready. She expired on January 26, about T P.M.

On the 28, I made a post mortem examination. The body had most rapidly decomposed. The face was sadly changed; the mouth, eyes, and nose, had turned to a dirty greenish hue; the fuetor was horrible; a quantity of dark bloody fluid escaped in profusion from the mouth and nostrils.

On opening the abdomen, to my surprise, I found no evidence of peritonitis; not a trace of lymph or pus was seen. The intestines, both large and small, and the stomach, were enormously distended with flatus, and otherwise nearly empty; the peritoneum was shining, here and there, perhaps, redder than usual. On the right side, about the cecum, and extending upwards to the liver, a bloody fluid was effused, of a tenacious viscid consistence, like treacle, only redder. By the side of the caput colj, I found a loose saccular body of the size of a child's head, extending upwards to the liver, and discoloured by contact with bile; it was rup. tured at its upper extremity, and a similar dark fluid was still flowing out. It proved to be a cyst, and was connected to the right side of the uterus, evidently ovarian and unultilocular. This had burst into the peritoneal cavity, and was the cause of all her sufferings. The uterus was normal, and contained a fine five months fuetus, the placenta completely adherent. The Fallopian tube was healthy; its fimbriated extremity was loose and patent, admitting an ordinary probe for several inches. A distinct and well-formed corpus luteum, with its cavity all but closed, was observed. The cyst, on examination, contained several smaller cysts within the larger envelope; some gelatinous and transparent; others filled with bloody, dark contents, and opaque. The coats of the cyst were thick and opaque near the ovary, and gradually became thinner and transparent towards the loose extremity; in fact, so thin that only a slight 
force would have easily ruptured it. The cyst and fœtus are in the museum of St. Bartholomew's Hos. pital.

In this case, the patient was ignorant of any abdominal tumour excepting pregnuncy, and, consequently, ho suspicion was entertained of the presence of an ovarian cyst. Had her medical advisers been aware of such a growth, I do not see what treatment could have been beneficial. The chances of recovery, supposing extirpation had been attempted, must have been very small, considering the great base of the connections of the cyst; while the occurrence of fatal collapse seems the must probable issue from any surgical interference. Death seems to have resulted from two causes: the prostration consequent upon the nervous shock; and the decomposition of the blood and other tissues that were effused into the peritoneal cavity, and their poisonous effects upon the system, It is singular that no products of inflammatory action were discovered, in the shape of lymph or purulent effusion. A slight increased redness was all I could discover; and this, perhaps, was not the effect of increased vascular action, but of those static changes by which the tissues are merely stained with the colouring matter of the blood.

Dr. Barnes has made some most instructive observations upon this case, in a clinical lecture published in the Lancet, Aug. 3, 1861, which will well repay an attentive perusal. He says, "From the extreme proneness of the body to decompose, it may be also conjectured that the blood had become poisoned, either by the absorption of fluid that escaped from the ruptured cyst, or by the disturbance of the blood-forming and blood-purifying functions induced by the shock."

CASE II. Nov. 4, 1860 . I was called to a married female, aged 48 , the mother of eight children, all healthy. She had never been ill, except during her confinements. Her parents were healthy; but her mother died, aged 63, of dropsy. She had been attended by a surgeon for an attack of diarrhœa, accompanied by pain in the right side, under the liver. There was loss of appetite, frequent purgings, with severe colicky pains, great thirst, general abdominal ten. derness, excessive flatulence, glazed red tongue, and feeble quick pulse. I thought there was indistinct fluc. tration immediately above the pubes; but the surgeon who had been in attendance had not noticed it, nor could ho now detect it.

Astringents and opiates gave relief; but she continued weak, with low typhoid symptoms, for some time. Her complexion was sallow. Stimulants were freely given with diuretics, as the urine was very scanty and darkcoloured, but contained no albumen. I examined the body more carefally, and was doubiful whether the fluctuation, which was still indistinct, was ascitic or ovarian. The body felt too tense and hard for mere ascites; I inclined, therefore, to the opinion that it was an ovarian cyst. Iodide of potassium, decoction of scoparium, and bark, were given with advantage.

Jan. 28. As the tumour increased, I sent her to my friend Dr. - , who kindly gave me his opinion as follows:-"I think the case ovarian; the dulness in front below the umbilicus, the clear sound above, the clear sound at the right iliac region, all go with encysted dropsy, and negative the impression of ascites. Moreover, the upper border of the tumour is very distinctly felt a little above the umbilicus. I fear there is some solid matter at the left side, though otherwise the cyst appears unilocular. I wish I could give you any useful suggestion for treatment. I fear that letting alone, iodine injection, and extirpation, are the three alternatives ; none very satisfactory."

June 8. Dr. - again saw the patient, and wrote: - The case seems to me one in which the question of extirpation may be very fairly entertained. There still seems to meless distinct fluctuation in the left iliac re. gion than elsewhere; but I should not think that there is any such presence of solid matter as to negative the operation, though it may, perhaps, require some increase in the size of the incision."

March 7. Mr. - saw her, and wrote as follows - I consider the case to be enlargement of the right ovary, partly solid and partly fluid. If the tumour in. creases, then I should advise its removal, which should be done either at her house or a hospital. In the meantime, I wish your treatment (the bark and iodide of po. tassium) to be pursued. P.S. I consider the case very favourable for operation."

June 16. Fluctuation was now very distinct in every direction. The body was hard, and the tumour extended above the umbilicus almost to the ribs towards the left hypochondrium. 'The health was now tolerably good ; but there had been profuse menorrhagia ; in fact, she was seldom free from discharge for more than a week or ten days at a time. Gallic acid, infusion of roses, and quinine, had been given to keep this in check. The os nteri was patent, and flabby, and large. She could walk several miles; and was willing to undergo an operation if advisable, notwithstanding the full dangers had been pointed out. She had never had a miscarriage; the catamenia had always been regular, but profuse. The operation was given up for the pre. sent, till she experienced more decided inconvenience from the tumour.

Aug. 12. Another eminent surgeon saw the patient with me. He gave me his opinion that there were no pelvic adhesions; that the os uteri was large and spongy; that the tumour was ovarian and multilocular; and observed that he could distinctly trace the boundaries of several cysts. He said that it would require a long incision of ten inches, as there was probably much solid matter; and that the case was favourable for operation. He did not advise it immediately; but, as her health was good, to wait till it interfered with the breathing, or caused other distressing symptoms. She applied turpentine every night, which, she persisted, had done much good.

Sept. 25. Her husband came for something to stop sickness and violent pain over the whole stomach. The bowcls were not confiued. Effervescing salines were given.

Sept. 26. I found her in bed, rolling from side to side in great pain, constantly vomiting a dark green fluid; everything taken returned immediately. There was slight chilliness, but no rigor. The skin was cool; palse slow and soft; tongue furred, and white in the centre, with red edges; the abdomen was tender, but she eould bear it to be fairly examined. Fluctuation was clear; there was no tympanitic condition. Calomel and opium, in grain doses, were given to relieve the pain and romiting. Turpentine stupes were applied to the abdomen. She was allowed barley-water only to drink.

Sept. 27. She slept well, and was easy througbout the night; but this morning she awoke with severe pain and romiting. The catamenia had been flowing for a fortnight, and had stopped; but since the vomiting, they yesterday reappeared. There was no fever; the abdo: men was not more tender. 'The calomel and opium pills were continued.

Sept. 28. The bowels were relieved by an enema. The pain and vomiting had ceased.

Oct. 3. She continued to improve up to yesterday, when vomiting returned, but without pain. The abdomen was very tense, tympanitic in the epigastrium and over the semicolon. 'The bowels had not been moved, though she had taken several doses of castor-oil. She lay in an apathetic dozing state, upon her back or left side, with her legs drawn up. She was perfectly conscious. The skin was cool and sweating; pulse small, under 80 ; there were great thirst, hiccup, and an anxious leaden countenance. She passed very little uriue, which had a slight trace of albumen. 
Oct. 4. She was evidently sinking; she was free from pain, but had occasional vomiting. The epigas. trium was very tympanitic. An enemá was tried, but the sphincter did not retain it. She expired, perfectly conscious, about 10.30 P.M.

Oct. 5, at 4 P.M., I made a post mortem examination The abdomen was very tense. The dimensions of the tumour could be plainly seen, bounded by the distended stomach above and semicolon on the left side. I dissected, as carefully as I possibly could, through the abdominal walls; and, on opening the peritoneum (proceeding as in ovariotomy), there escaped in a jet about a gallon of perfectly clear limpid serum. I was doubtful now whether the fluid was ascitic, or that the cyst adhered to the abdominal walls. A probe was introduced, but no adhesions could be felt. On extending the incision upon a director, it seemed that the cavity. containing the fluid filled the lower half of the abdomen from the rim of the pubes to about two inches above the umbilicus. There lay collapsed, at the bottom of this cavity, a large gelatinous cyst, floating in the fluid. It was perfectly white, and tore so readily as not to bear its own weight. It was formed of albuminous jelly-like layers, with a granular internal surface, and perfectly smooth exterior, and was as large as a child's head, and pyramidal in shape. It had evidently burst into the larger cavity, and floated loose, having no point of adhesion.

On further examination, the larger cavity was found to be a large cyst, everywhere adherent to the peritoneum, with numerous bands or bridles running in different directions from its posterior surface towards the mesentery, intestines, and other viscera. It was firmly adherent, and required the greatest care to dissect it from its connections; but here and there small patches of smooth unattached peritoneal membrane were traced with the corresponding peritoneal surface of the intestines facing, proving it to be an ovarian cyst. It had evidently started from the right ovary, forming attach. ments to the under surface of the fundus uteri, thence to the posterior surface of the bladder, extending up. wards in contact with the abdominal walls, and pushing the intestines backward, and firmly adhering to them. It could admit of no further distension, owing to its connections, confining the uterus and bladder, so that they could not be raised above the pubes. The fundus of the bladder might be seen shining through an oval patch, of about the size of a crown-piece, where the cyst was perfectly transparent and very thin, as if it had expanded in this direction. The left ovary was free; the fimbriated extremity of each ovary was loose and highly congested. The uterus was hard, as large as an orange, filied with a coagulum of blood. The os was of a dark purple colour, soft and velvety; the cervix hard and enlarged, admitting a director with some difficulty, Had impreguation taken place, the uterus must have thrown off the ovum at an early period, for the uterus could not have risen above the pelvic brim. The bladder also, from the same cause, could not have contained an ordinary quantity of urine, and she was obliged throughout to pass it very often. All the other viscera were bealthy. The cyst was decidedly unilocular, and no solid matter contributed to its size.

The above case shows how the subject of ovariotomy is beset with difficulties. No one will surely, after the post mortem disclosures, think that extirpation could have been practicable in this case. During life, the diagnosis of several gentlemen, admitted to be authorities on the question of ovarian disease, was, that the removal of the cyst by operation was favourable, On this point, all seemed agreed, and that the amount of solid matter was considerable. Mr. Wells only considered it to be multilocular. It seems that tapping and injection by iodine might have been practised with the greatest hope of success; and, owing to the complete adhesions, no fluid could have escaped into the 172 abdominal cavity, and the chances of peritoneal inflam. mation therefore were much diminished.

I have published the above case, not to discourage ovariotomists, but to show how great caution is needed before proceeding to extirpation. If the most experienced fail in the question of diagnosis, it surely behoves the humbler disciple to pause long before he undertakes an operation, the expediency of which is too broadly condemned by some, while it is, on the othen hand, too indiscriminately addopted by others.

\section{THE GRANULATION OF MEDICINES.}

By Thomas Skinner, M.D., Physician to the Liverpool Dispensaries; Fellow of the Obstetrical Society of London, etc.

" By the application of art, it is intended that medi. cines should be rendered more agreeable, more convenient, more safe, and more efficacious than they are in their natural state. To obtain these ends is the intention of pharmacy." (Duncan's Elements of Therapeutics, 1773, volume i.)

In furtherance of the above elegant and forcibly ex. pressed ideas of Duncan, I beg to submit the following as an addition, if not an improvement, to the methodus medendi: namely, the Granulation of Medicines.

In an admirable paper by Dr. Henry Kenvedy, of Dublin, which appeared in the pages of the Dublin Medical Press, and an abstract of which appeared shortly afterwards in the first volume of this year's JouRnaL ( $p$. 524 ), it is laid down by him as an axiom in therapeutics, that the form of powder "is the most efficacious in which a medicinal substance can be administered." Having always entertained the same opinion myself, I have frequently thought of some method which would render powders more easy of deglutition, and less offensive to the senses of taste and smell. It at once occurred to me that the granular form, with sugar or gum as the excipient, would suit the purpose; but how to obtain the granular form without having recourse to the art of the confectioner, or without the aid of an excessive amount of heat, sadly puzzled me. On explaining $\mathrm{my}$ difficulties to Mr. Samuel Banner, an enterprising chemist in this town, and at the same time informing him that the French pharmaceutists had a form of medication which they call "poudres granulees," the process of preparing which consisted in enveloping the particles of medicines in syrup by means of heat and constant stirring, as in the art of making comfits, he at once suggested a very simple plan, by means of which almost every medicine capable of assuming the form of powder may be granulated of a firm consistence; a process which demands little or no aid from heat; which requires very little moisture, and which results in a granular powder that may be laid upon the tongue and washed over the throat by means of water, without leaving a trace of a medicinal substance, or the slightest sensation to indicate its recent proximity to the peripheral extremities of the gustatory and olfactory nerves. The process is a modification of the method of granulating gunpowder.

Method of Preparing the Granules. The first requisite is a thoroughly good material; to obtain which, it is necessary that all powders, previously to their being converted into granules, should be fresh and of the best quality. As regards pulverisation, it may be as well to observe, that in order to form granules it is not necessary that the powder should be impalpably fine; a coarsely ground powder being quite as easily granulated as an impalpable one, and the resulting granule being quite as efficacious, if not more so, for reasons which will hereafter appear.

1. Making the Mass. The powder is to be placed in a Wedgwood mortar, and sufficient mucilage of gum arabic, of the London or Edinburgh Pharmacopeia 